\title{
Gut Faecalibacterium may improve impaired tacrolimus metabolism in kidney transplant recipients with cytochrome polymorphism
}

\author{
Ji Eun Kim ${ }^{1}$, Hyo-Eun Kim², Hyunjeong Cho ${ }^{3}$, Ji In Park ${ }^{4}$, Jang Wook Lee ${ }^{2}$, Seung Hee Yang ${ }^{2}$, Jung Pyo Lee ${ }^{5}$, Jongwon Ha ${ }^{6}$, \\ Yon Su Kim², Hajeong Lee ${ }^{2}$
}

\footnotetext{
${ }^{1}$ Division of Nephrology, Department of Internal Medicine, Korea University Guro Hospital, Seoul, Korea

${ }^{2}$ Division of Nephrology, Department of Internal Medicine, Seoul National University Hospital, Seoul, Korea

${ }^{3}$ Division of Nephrology, Department of Internal Medicine, Chungbuk National University Hospital, Cheongju, Korea

${ }^{4}$ Division of Nephrology, Department of Internal Medicine, Kangwon National University Hospital, Chuncheon, Korea

${ }^{5}$ Division of Nephrology, Department of Internal Medicine, SMG-SNU Boramae Medical Center, Seoul, Korea

${ }^{6}$ Division of Transplantation, Department of Surgery, Seoul National University Hospital, Seoul, Korea
}

Background: The gut microbiota alters expression of drug-metabolizing enzymes and transporters, and consequently affects therapeutic dose and response of medicines. We aimed to investigate the effect of the gut microbiome on the variation of blood tacrolimus concentration in kidney transplant (KT) recipients.

Methods: Metagenomes in pre- and post-transplant stool samples of KT recipients were analyzed by the Illumina MiSeq. Tacrolimus trough levels from every recipient were obtained for 1 year after KT. Additionally, archival peripheral blood of recipients was tested for CYP3A5 genotyping by SNaPshot assay.

Results: A total of 77 and 60 fecal samples from pre- and post-transplantation were analyzed from 77 recipients, respectively. The mean age was $48.4 \pm 12.0$ years, and $59.7 \%$ was male. The recipients were classified as low- and high-variability dose groups by time-series k-means clustering through dose-adjusted concentrations of tacrolimus in 1 year. High-variability group showed higher mean $(90.2 \pm 32.5$ vs. $192.8 \pm 40.1, P<0.001)$ and standard deviation $(41.4 \pm 21.9$ vs. $110.7 \pm 34.8, P<0.001)$ of tacrolimus level compared to the low-variability group, respectively. Interestingly, only $56.5 \%$ of CYP3A5 nonexpresser (CYP3A5*3/*3) was included in low-variability group while $100 \%$ of CYP3A5 expressors (CYP3A $5 * 1 / * 1$ or CYP3A $5 * 1 / * 3$ ) were included in low-variability group. In order to reveal this individual difference of tacrolimus variability in CYP3A5 nonexpresser, we analyzed fecal microbiota in CYP3A5 nonexpresser and found significant inverse relationship between dose-adjusted tacrolimus level and relative abundance of Faecalibacterium in post-transplant fecal samples $(P=0.018)$. In multivariable logistic analysis adjusted by age, sex and major genera, the elevation of Faecalibacterium in post-transplant significantly reduced the risk of high-variability group (odds ratio [OR], 0.64; 95\% confidence interval [CI], 0.44-0.93; $P=0.018$ ), while the statistical significance was diminished after further adjustment of genotypes (OR, $0.46 ; 95 \% \mathrm{Cl}, 0.21-1.01 ; \mathrm{P}=0.053)$.

Conclusions: Gut Faecalibacterium influences the metabolism of tacrolimus, and it may improve impaired metabolism of tacrolimus in CYP3A5 nonexpresser. Further studies on the mechanism of Faecalibacterium on tacrolimus levels are needed.

Corresponding author: Hajeong Lee

E-mail: mdhjlee@gmail.com

(c) The Korean Society for Transplantation

This is an Open Access article distributed under the terms of the Creative Commons Attribution Non-Commercial License (http://creativecommons.org/licenses/by-nc/4.0/) which permits unrestricted non-commercial use, distribution, and reproduction in any medium, provided the original work is properly cited. 